\title{
Metallicity Differences in Type la Supernova Progenitors Inferred From Ultraviolet Spectra
}

\section{Citation}

Foley, Ryan J., and Robert P. Kirshner. 2013. "METALLICITY DIFFERENCES IN TYPE la SUPERNOVA PROGENITORS INFERRED FROM ULTRAVIOLET SPECTRA." The Astrophysical Journal 769 (1): L1. https://doi.org/10.1088/2041-8205/769/1//1.

\section{Permanent link}

http://nrs.harvard.edu/urn-3:HUL.InstRepos:41412198

\section{Terms of Use}

This article was downloaded from Harvard University's DASH repository, and is made available under the terms and conditions applicable to Other Posted Material, as set forth at http:// nrs.harvard.edu/urn-3:HUL.InstRepos:dash.current.terms-of-use\#LAA

\section{Share Your Story}

The Harvard community has made this article openly available.

Please share how this access benefits you. Submit a story.

Accessibility 


\title{
METALLICITY DIFFERENCES IN TYPE Ia SUPERNOVA PROGENITORS INFERRED FROM ULTRAVIOLET SPECTRA
}

\author{
Ryan J. Foley AND RoBert P. KIRSHNER \\ Harvard-Smithsonian Center for Astrophysics, 60 Garden Street, Cambridge, MA 02138, USA \\ Received 2013 February 18; accepted 2013 April 6; published 2013 May 1
}

\begin{abstract}
Two "twin" Type Ia supernovae (SNe Ia), SNe 2011by and 2011fe, have extremely similar optical light-curve shapes, colors, and spectra, yet have different ultraviolet (UV) continua as measured in Hubble Space Telescope spectra and measurably different peak luminosities. We attribute the difference in the UV continua to significantly different progenitor metallicities. This is the first robust detection of different metallicities for SN Ia progenitors. Theoretical reasoning suggests that differences in metallicity also lead to differences in luminosity. SNe Ia with higher progenitor metallicities have lower ${ }^{56} \mathrm{Ni}$ yields and lower luminosities for the same light-curve shape. $\mathrm{SNe}$ 2011 by and 2011 fe have different peak luminosities $\left(\Delta M_{V} \approx 0.6 \mathrm{mag}\right)$, which correspond to different ${ }^{56} \mathrm{Ni}$ yields: $M_{11 \mathrm{fe}}\left({ }^{56} \mathrm{Ni}\right) / M_{11 \mathrm{by}}\left({ }^{56} \mathrm{Ni}\right)=1.7_{-0.5}^{+0.7}$. From theoretical models that account for different neutron-to-proton ratios in progenitors, the differences in ${ }^{56} \mathrm{Ni}$ yields for SNe 2011 by and $2011 \mathrm{fe}$ imply that their progenitor stars were above and below solar metallicity, respectively. Although we can distinguish progenitor metallicities in a qualitative way from UV data, the quantitative interpretation in terms of abundances is limited by the present state of theoretical models.
\end{abstract}

Key words: supernovae: general - supernovae: individual (SN 2011by, SN 2011fe)

Online-only material: color figures

\section{INTRODUCTION}

Type Ia supernovae (SNe Ia) are extremely useful for measuring cosmic distances because they are intrinsically luminous, and after standardization (e.g., Phillips 1993), have very small scatter in their intrinsic luminosities. Because of these qualities, $\mathrm{SNe}$ Ia were used to discover the Universe's acceleration (Riess et al. 1998; Perlmutter et al. 1999) and are useful for measuring cosmological parameters (e.g., Suzuki et al. 2012). These measurements assume that SNe Ia across all redshifts, and thus cosmic time, have the same luminosity after standardization by light-curve shape.

Recent observations suggest ways to improve this paradigm. $\mathrm{SNe}$ Ia in late-type host galaxies have systematically larger peak luminosities than those in early-type galaxies (e.g., Hamuy et al. 1996). Those differences mostly disappear after correcting for light-curve shapes. But even after that correction, there is a slight difference in the luminosities of SNe Ia coming from high-mass, passive host galaxies and low-mass, star-forming galaxies (Kelly et al. 2010; Lampeitl et al. 2010; Sullivan et al. 2010). This effect is small but real, amounting to a difference of $\sim 0.08 \mathrm{mag}$ compared to the $\sim 0.2$ mag effect of acceleration relative to a matter-only universe at $z \approx 0.5$. Even with the lowest scatter measurements (e.g., Mandel et al. 2011) and correcting for additional parameters (e.g., Foley \& Kasen 2011), intrinsic scatter remains. Present methods for correcting luminosities do not account for all the physical diversity among SNe Ia.

Additionally, SNe Ia from progenitor systems with winds or outflows tend to have higher ejecta velocities than those that have no indication of winds or outflows (Foley et al. 2012c). This observational difference may presage variance in peak luminosity. Other subtle differences in the observables may relate to the SN progenitor system. Timmes et al. (2003) predicted that $\mathrm{SNe}$ Ia from progenitors that differ by metallicity alone will produce different amounts of ${ }^{56} \mathrm{Ni}$, and emit different peak luminosities. Higher metallicity results in additional neutrons, which produce more stable Fe-group elements and less radioactive ${ }^{56} \mathrm{Ni}$. Mazzali \& Podsiadlowski (2006) found that altering the progenitor metallicity changes the peak luminosity of $\mathrm{SNe}$ Ia, but it would not change the light-curve shape. As a result, the light-curve shape correction would be insufficient to perfectly calibrate SN Ia luminosities, and would result in increased scatter. If progenitor metallicity changes (on average) with redshift, SN Ia distances will be systematically biased with redshift.

Theoretical studies indicate that varying the metallicity of an SN Ia progenitor does not significantly affect the optical spectral energy distribution (SED) of an SN Ia, but will dramatically change the UV SED (e.g., Höflich et al. 1998; Lentz et al. 2000; Sauer et al. 2008; Walker et al. 2012). Increased progenitor metallicity changes the final composition of the SN ejecta and increases the line blanketing in the UV. As a result, we expect a correlation between the UV SED of an SN Ia and its peak luminosity.

There have also been several observational efforts to obtain UV spectra of SNe Ia (e.g., Jeffery et al. 1992; Foley et al. 2008, 2012a; Sauer et al. 2008; Maguire et al. 2012). However, most spectra from these studies do not probe below $2500 \AA$, have a poor signal-to-noise ratio $(\mathrm{S} / \mathrm{N})$, or were obtained significantly after maximum brightness. The exceptions are SNe 2011by (presented here), 2011fe (Foley 2012), and 2011iv (Foley et al. 2012b), which all have excellent maximum-light UV spectra obtained by the Hubble Space Telescope (HST).

In this Letter, we forge the observational link between the UV SED of SNe Ia and their peak luminosities, confirming the theoretical expectation. We examine HST STIS spectra of two SNe Ia: SNe 2011 by and 2011fe. We present these data in Section 2. Besides SN $2011 \mathrm{iv}$, these are the only maximum-light spectra of SNe Ia with a reasonable $\mathrm{S} / \mathrm{N}$ that probe wavelengths $<2800 \AA$ A. Conveniently, both SNe have negligible host-galaxy reddening and nearly identical optical colors, light-curve shapes, and spectra. In Section 3, we show that the SNe have different UV spectra, which implies that the progenitor of SN 2011by had 

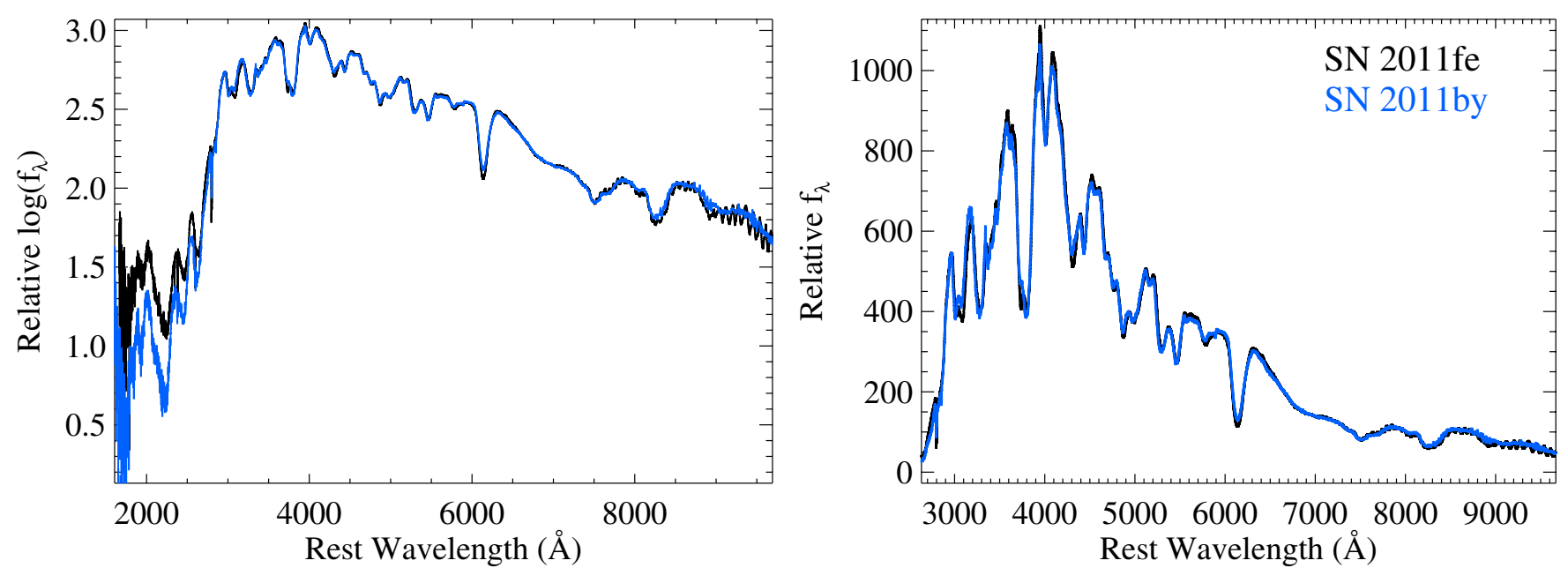

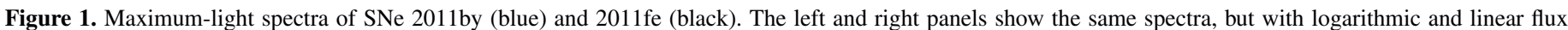
scales, respectively, and slightly different wavelength ranges.

(A color version of this figure is available in the online journal.)

a higher metallicity than SN 2011fe. In Section 4, we discuss the implications of this result.

\section{OBSERVATIONS AND DATA REDUCTION}

SN 2011by was discovered by Jin \& Gao (2011) on 2011 April 26.8 (UT used throughout) in NGC 3972, an Sbc galaxy with $D=18.5 \mathrm{Mpc}(\mu=31.34 \pm 0.36 \mathrm{mag})$ from a Tully-Fisher measurement (Tully et al. 2009). Zhang et al. (2011) obtained an optical spectrum of SN 2011by on 2011 April 27.5 , only 0.7 days after discovery, and determined that it was a young SN Ia.

Silverman et al. (2013) published optical light curves and spectra. The SN is spectroscopically normal with minimal dust reddening. Silverman et al. (2013) determined that SN 2011by reached maximum brightness in the $B$ band on 2011 May 9.9 and had $\Delta m_{15}(B)=1.14 \pm 0.03$ mag. Maguire et al. (2012) reported that SN 2011by peaked on 2011 May $9.6 \pm 0.1$. Throughout this Letter, we use the Maguire et al. (2012) value. Johansson et al. (2013) presented Herschel data which indicate that there was a minimal amount of circumstellar dust $\left(M_{\text {dust }} \lesssim 0.1 M_{\odot}\right)$.

SN 2011by was observed by HST using the STIS spectrograph (Program GO-12298; PI: Ellis) on 2011 May 9.36, corresponding to $t=0.1$ days relative to $B$ maximum. The spectra were obtained with two different gratings and the $52^{\prime \prime} \times 0^{\prime \prime} \cdot 2$ slit. Two exposures were obtained for each of the MAMA/ G230L and CCD/G430L setups with total exposure times of 5316 and $2263 \mathrm{~s}$, respectively. The two setups yield a combined wavelength range of 1605-4695 $\AA$. We retrieved the data from the Mikulski Archive for Space Telescopes. The data were reduced using the standard HST Space Telescope Science Data Analysis System (STSDAS) routines to bias subtract, flat-field, extract, wavelength-calibrate, and flux-calibrate each SN spectrum. Maguire et al. (2012) presented only the G430L spectrum. We combine the HST spectrum with an optical spectrum obtained less than one day later (on 2011 May 10.24) from Silverman et al. (2013). This extends the spectrum to $10196 \AA$.

SN $2011 \mathrm{fe}$ was discovered by the Palomar Transient Factory (Nugent et al. 2011a) in M101, an Scd at $D=6.4 \mathrm{Mpc}$ $(\mu=29.04 \pm 0.05 \mathrm{mag}$; Shappee \& Stanek 2011). Various studies (e.g., Li et al. 2011; Nugent et al. 2011b; Bloom et al. 2012; Horesh et al. 2012; Johansson et al. 2013; Margutti et al. 2012; Patat et al. 2013) all indicate that SN 2011fe had negligible circumstellar and interstellar dust reddening and the companion star was either a white dwarf or a low-mass nondegenerate star. Several groups have determined that SN $2011 \mathrm{fe}$ had $\Delta m_{15}(B)=1.10$ mag (e.g., Richmond \& Smith 2012). An HST spectrum of SN 2011fe (Program GO-12298; PI Ellis) with a phase of $t=0.0$ days relative to $B$-band maximum brightness was presented by Foley (2012). We examine this spectrum further here.

The spectra were dereddened by the Galactic values of Schlafly \& Finkbeiner (2011): $E(B-V)=0.012$ and $0.008 \mathrm{mag}$ for SNe 2011 by and 2011fe, respectively. We display the spectra in Figure 1.

\section{ANALYSIS}

SNe 2011by and 2011fe have very similar light curves (with $\Delta m_{15}(B)=1.14$ and $1.10 \mathrm{mag}$, respectively, and indistinguishable rise times; see Figure 2). The $\mathrm{SNe}$ also have identical observed colors. Using the Silverman et al. (2013) data, we find that SN 2011by peaked at $V=12.91 \pm 0.01 \mathrm{mag}$, while SN 2011fe peaked at $V=9.99 \pm 0.01 \mathrm{mag}$ (Richmond \& Smith 2012). Correcting for Milky Way extinction and using our adopted distance moduli, SNe 2011by and $2011 \mathrm{fe}$ peaked at $M_{V}=-18.47 \pm 0.36$ and $-19.07 \pm 0.05 \mathrm{mag}$, respectively. These values are only $1.7 \sigma$ different, but if the distances are correct, SN 2011 by is $\sim 0.6$ mag fainter at peak than SN $2011 \mathrm{fe}$. It appears that SN 2011by has a low peak luminosity for its light-curve shape.

The $\mathrm{SNe}$ also have indistinguishable ejecta velocities $\left(v_{\mathrm{Si} \text { II }}^{0}=\right.$ $-10,300 \pm 200$ and $-10,400 \pm 200 \mathrm{~km} \mathrm{~s}^{-1}$, respectively). Figure 1 demonstrates that these two spectra are virtually identical in the optical. However, the spectra diverge at wavelengths $<2700 \AA$, with SN $2011 \mathrm{fe}$ having more UV flux.

Despite the clear similarity in the optical, we investigate if dust reddening can cause the UV differences. To make the UV spectra match, we must assume an extinction of $A_{V}=0.5 \mathrm{mag}$ with $R_{V}=3.1$ and a Cardelli et al. (1989) reddening law modified by O'Donnell (1994; Figure 3). However, doing this makes the near-UV and optical continua significantly different. A non-standard $R_{V}$ cannot account for the difference. The best explanation is that SNe 2011by and 2011fe have similar (and 


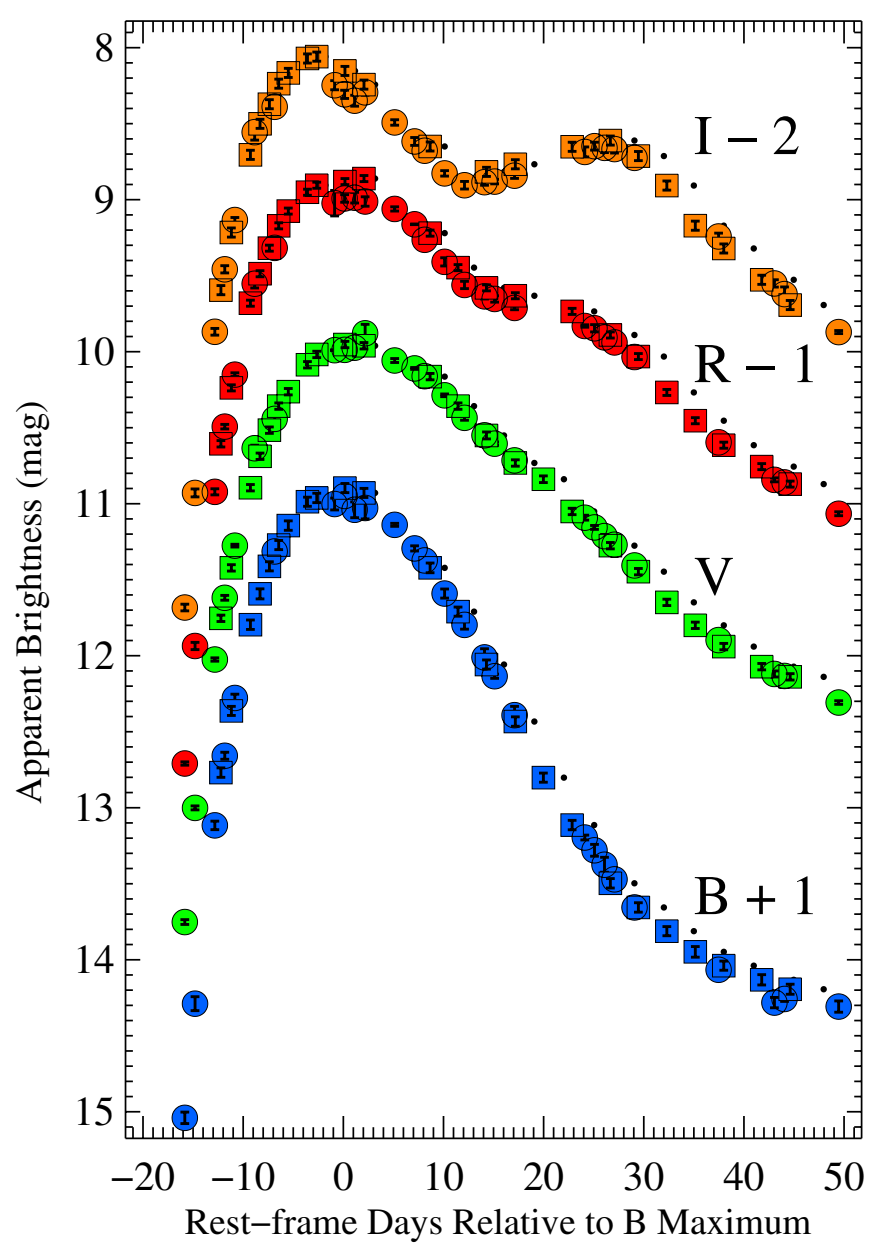

Figure 2. $B V R I$ light curves of SNe 2011by (Silverman et al. 2013, squares and points) and $2011 \mathrm{fe}$ (Richmond \& Smith 2012, circles) with offsets noted. Both SNe have been corrected for Galactic extinction. SN 2011by has been shifted by $-2.91 \mathrm{mag}$ in all bands to match the apparent peak magnitudes of SN $2011 \mathrm{fe}$, which corresponds to a peak absolute magnitude difference of $0.6 \mathrm{mag}$. The black points are the raw SN 2011 by light curves. The squares are the result of shifting the time of maximum $B$ brightness by +1 day and stretching the light curve by a factor of 0.95 , consistent with the difference in light-curve shape. Any differences between the light curves can be attributed to inaccurate times of maximum, slightly different light-curve shapes, and slightly different filter responses (Stritzinger et al. 2002).

(A color version of this figure is available in the online journal.)

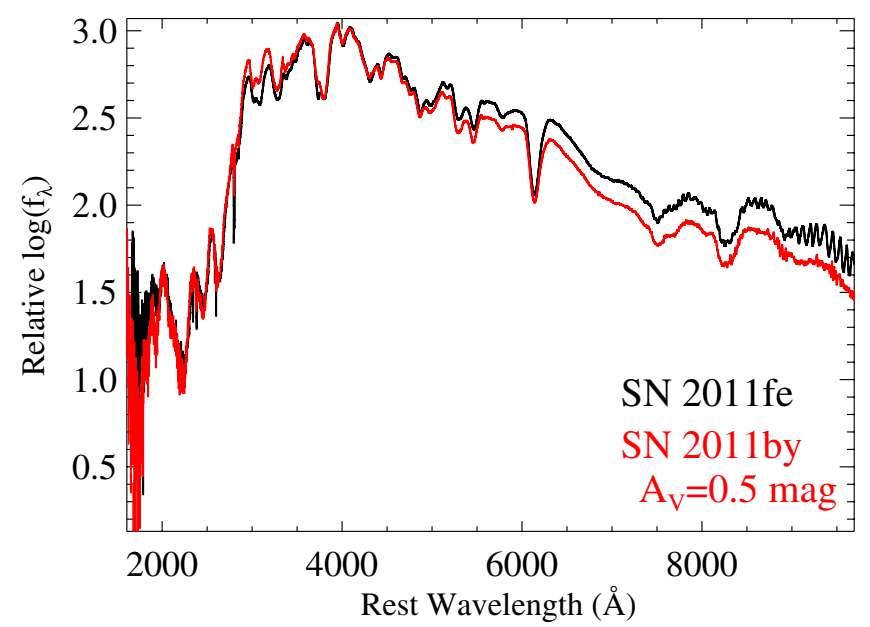

Figure 3. Maximum-light spectra of SNe 2011by (red) dereddened by $A_{V}=$ $0.5 \mathrm{mag}$ and $R_{V}=3.1$ and $2011 \mathrm{fe}$ (black).

(A color version of this figure is available in the online journal.)

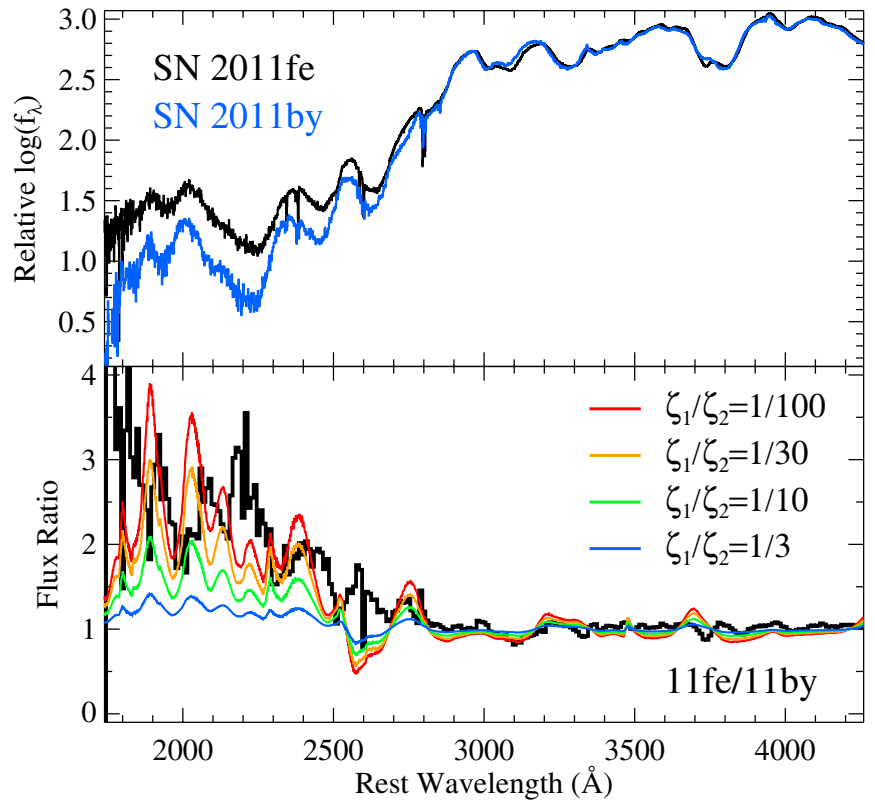

Figure 4. Top: maximum-light spectra of SNe 2011 by (blue) and 2011 fe (black). Bottom: flux-ratio spectra for these SNe in black. Overplotted are the average flux-ratio spectra of the Lentz et al. (2000) $\tau=15$ day spectra, with differences in the metallicity factors, $\zeta$, labeled.

(A color version of this figure is available in the online journal.)

probably negligible) host-galaxy dust reddening, but physical differences that create the differences in the UV SEDs.

In Figure 4, we compare the UV spectra of the SNe against each other and examine the flux ratio of the spectra (having scaled the $\mathrm{SNe}$ to match in the optical, and having the same scalings as shown in Figure 1).

Since SNe 2011fe and 2011by have very similar light curves and ejecta velocities, the differences in their far-UV spectra must be uncorrelated with these parameters and related to another parameter. Similarly, the cause of the UV difference must not affect the optical properties of the SN. For example, mixing of freshly synthesized $\mathrm{Fe}$-group material to the outer layers should also mix out ${ }^{56} \mathrm{Ni}$, which should change the rise time. One viable candidate to produce the UV difference is progenitor metallicity.

Lentz et al. (2000) generated several model SN Ia spectra that cover UV wavelengths with varying metallicity. Starting with a single W7 model, they changed the outer-layer metallicity for elements heavier than $\mathrm{O}$ by factors of $\zeta=1 / 30,1 / 10,1 / 3$, 1 , and 3. Increasing the progenitor metallicity depressed the UV flux. Höflich et al. (1998) found the opposite trend, but this was the result of differing density structures (Lentz et al. 2000). Although the method of Lentz et al. (2000) is far from a complete treatment of how metallicity affects the spectra of $\mathrm{SNe}$ Ia, the models provide some predictions for short wavelengths.

Although the models generally match the data, they do not precisely reproduce all spectral features. By using flux ratios, we avoid some of the uncertainties in matching the data to the models. Flux-ratio spectra focus on the differences between models, which is metallicity for the Lentz et al. (2000) models. The flux ratios of models with the same ratio of metallicity factors (i.e., comparing the $\zeta=1 / 30$ and $1 / 3$ models and the $\zeta=1 / 10$ and 1 models) all have similar shapes and amplitude. This means that the flux-ratio spectra, in principle, can determine differences in metallicity even if the absolute metallicity is not well determined. For each ratio of metallicity factors $\left(\zeta_{1} / \zeta_{2}=1 / 3,1 / 10,1 / 30\right.$, and $\left.1 / 100\right)$, we produced an 
average flux-ratio spectrum and present the spectra in Figure 4. Although the flux ratios remove some of the uncertainties from the models, more realistic models are required to produce higher fidelity results.

The model flux-ratio spectra are all approximately 1 (i.e., the individual spectra are similar) for $\lambda>2500 \AA$. Blueward of $2500 \AA$, the lower metallicity models have an UV excess relative to the higher metallicity models. Moreover, the flux excess increases with larger differences in metallicity. For a metallicity factor ratio of $1 / 3$, the flux difference is relatively small and less than $40 \%$ different at all wavelengths examined.

Some Lentz et al. (2000) flux-ratio spectra roughly match the SN 2011fe/SN 2011by flux-ratio spectrum. Ascribing the differences in the UV spectra to differences in progenitor metallicity, the progenitor of SN 2011 by had a higher metallicity than that of SN 2011fe. If the Lentz et al. (2000) models accurately reproduce the change in UV continuum for a given difference in metallicity, then the progenitor of SN 2011by had $>30$ times the abundance for elements heavier than O than SN 2011fe. This can be written as

$$
[\mathrm{O} / \mathrm{Fe}]_{11 \text { by }} \lesssim[\mathrm{O} / \mathrm{Fe}]_{11 \mathrm{fe}}+1.5
$$

Timmes et al. (2003) found that the ${ }^{56} \mathrm{Ni}$ yield of an SN Ia should depend on the metallicity of the progenitor star, deriving a simple equation relating the progenitor metallicity to the ${ }^{56} \mathrm{Ni}$ mass,

$$
M\left({ }^{56} \mathrm{Ni}\right) \propto 1-0.057 Z / Z_{\odot} .
$$

For subsolar metallicity, this function is relatively flat. As an example, going from $[\mathrm{O} / \mathrm{Fe}]=-1.25$ to 0.25 or -0.7 to 0.8 (both $\Delta[\mathrm{O} / \mathrm{Fe}]=1.5$ ) would result in ${ }^{56} \mathrm{Ni}$ mass differences of $10 \%$ or $50 \%$, respectively. Large differences in ${ }^{56} \mathrm{Ni}$ mass can only occur if at least one progenitor had above solar metallicity.

Arnett (1982) showed that the peak bolometric luminosity of an $\mathrm{SN}$ Ia is directly proportional to the amount of ${ }^{56} \mathrm{Ni}$ generated in the explosion. Examining their light curves, SNe 2011by and $2011 \mathrm{fe}$ have consistent rise times. From their maximum-light spectra, they have the same optical and near-UV colors. With the reasonable assumption that SNe 2011by and 2011fe have the same bolometric corrections, we have

$$
\frac{M_{11 \mathrm{fe}}\left({ }^{56} \mathrm{Ni}\right)}{M_{11 \mathrm{by}}\left({ }^{56} \mathrm{Ni}\right)}=10^{0.4\left(M_{V, 11 \mathrm{by}}-M_{V, 11 \mathrm{fe}}\right)},
$$

and thus $M_{11 \mathrm{fe}}\left({ }^{56} \mathrm{Ni}\right) / M_{11 \mathrm{by}}\left({ }^{56} \mathrm{Ni}\right)=1.7_{-0.5}^{+0.7}$. The uncertainty in the distance to NGC 3972, the host galaxy of SN 2011by, is the largest uncertainty in the ${ }^{56} \mathrm{Ni}$ ratio; better measurements should improve our understanding of these SNe. Nonetheless, SN 2011fe generated more ${ }^{56} \mathrm{Ni}$ than SN 2011by, consistent with SN 2011fe having lower progenitor metallicity.

If the entire difference is attributed to metallicity, that provides an estimate for the progenitor metallicity. Using Equation (2) and the $1 \sigma$ lower bound of the ${ }^{56} \mathrm{Ni}$ ratio, corresponding to 1.2 , the progenitor of SN 2011 by must have been $4 Z_{\odot}$ if the progenitor of SN 2011fe had solar metallicity. That is, the progenitor of SN 2011by almost certainly had a metallicity above solar. From Equations (1) and (2), the best estimate of the ${ }^{56} \mathrm{Ni}$ ratio corresponds to metallicities of $[\mathrm{O} / \mathrm{Fe}]=-0.6_{-0.3}^{+0.1}$ and $0.9_{-0.3}^{+0.1}$ for SNe 2011 by and $2011 \mathrm{fe}$, respectively. These estimates rely on the Lentz et al. (2000) models, and better models may yield different values.

\section{DISCUSSION AND CONCLUSIONS}

We presented HST maximum-light UV spectra of two SNe Ia. SNe 2011 by and 2011 fe are "twin" SNe, having nearly identical light curves and optical spectra. SN 2011fe has a higher continuum at $\lambda<2500 \AA$ than SN 2011 by and was more luminous. Considering their remarkable similarity in other respects, the differences in peak luminosity and UV flux are likely connected. The physical cause of both effects must not affect other observables such as rise time and the optical spectrum. The only known way to change both the UV flux and the peak luminosity without affecting other observables is differing progenitor metallicity.

Comparing the flux-ratio spectrum of SNe 2011by and 2011fe to model flux-ratio spectra, we determined that SN 2011 by had a higher metallicity with $\Delta[\mathrm{O} / \mathrm{Fe}] \gtrsim 1.5$. The SNe had peak absolute magnitudes that differed by $\sim 0.6 \mathrm{mag}$, which corresponds to a ratio in their ${ }^{56} \mathrm{Ni}$ masses of $1.7_{-0.5}^{+0.7}$; the largest contribution to the uncertainty is from the distance to SN 2011by. Given the relative metallicity difference and ${ }^{56} \mathrm{Ni}$ mass ratio, we estimate that the progenitors of SNe 2011by and 2011fe had supersolar and subsolar metallicities, respectively. Improved modeling that includes more realistic physics (e.g., multi-dimensional models, improved flame physics) will be necessary to accurately and precisely measure progenitor metallicities.

SNe 2011 by and 2011 fe were hosted by Sbc and Scd galaxies which have gas-phase metallicities of $12+\log (\mathrm{O} / \mathrm{H})=8.97$ and 9.12 from Sloan Digital Sky Survey spectra (Prieto et al. 2008, using a solar value of 8.86), respectively. However, Bresolin (2007), performing a more detailed analysis of M101, found a metallicity of 8.93 (using a solar value of 8.66). Using the Bresolin (2007) metallicity and metallicity gradient, Stoll et al. (2011) determined that M101 had a metallicity of 8.45 at the position of SN 2011fe. A similar measurement must be made for SN 2011by, but taken at face value, the progenitor sites of the two SNe could differ in metallicity by $\sim 0.5$ and in the direction we found from the SNe. The metallicity at the radius of the $\mathrm{SN}$ may roughly indicate the progenitor metallicity.

There are three other reported measurements for SN Ia progenitor metallicity, but there is no differential metallicity measurement similar to what we have presented here. On the face of it, Taubenberger et al. (2008) found that the progenitor of SN 2005bl, a low-luminosity SN Ia, had subsolar metallicity based on the strength of optical Fe lines. By measuring the relative flux of X-ray lines from their SN remnants, Badenes et al. (2008) and Park et al. (2013) determined that the progenitors of Tycho's and Kepler's SNe both had $Z \approx 3 Z_{\odot}$ with relatively large uncertainties. It appears that the progenitors of SNe 2005bl and $2011 \mathrm{fe}$ had similar (subsolar) metallicities, while the progenitors of SN 2011by, Tycho's SN, and Kepler's SN had similar (supersolar) metallicities.

Using the SNLS sample of SNe Ia and proxies for ${ }^{56} \mathrm{Ni}$ mass (peak SN luminosity in multiple bands) and metallicity (hostgalaxy luminosity), Howell et al. (2009) also found a trend between the two quantities consistent with the predictions of Timmes et al. (2003). Similarly, several authors have found that $\mathrm{SNe}$ Ia in more massive galaxies have higher luminosity (after light-curve shape corrections) than those in less massive galaxies (Kelly et al. 2010; Lampeitl et al. 2010; Sullivan et al. 2010). These results are consistent with those found here, where the SN with a higher metallicity progenitor, SN 2011by, was fainter than the SN with the same light-curve shape but lower metallicity progenitor, SN $2011 \mathrm{fe}$. 
The average metallicity of SN Ia progenitors must increase with cosmic time and decreasing redshift. This, in turn, should produce fainter $\mathrm{SNe}$ for the same light-curve shape. Not accounting for this effect could bias SN Ia cosmological measurements. Specifically, SNe Ia at high redshift should have, on average, lower metallicity and higher luminosity than local comparison $\mathrm{SNe}$, resulting in an underestimate of the effect of acceleration. Using host-galaxy mass as a proxy for progenitor metallicity may remove this potential systematic bias. The effect of progenitor metallicity is relatively small, but accurately constraining the properties of dark energy demands attention to small systematic effects.

Supernova research at Harvard is supported by NSF grant AST-1211196. We thank the KITP for hospitality, where this research was supported in part by NSF grant PHY11-25915.

We thank W. Hillebrandt, M. Kromer, T. Piro, J. Silverman, M. Stritzinger, and S. Taubenberger for useful discussions, the anonymous referee for helpful comments, and J. Silverman for providing data.

This research has made use of the NASA/IPAC Extragalactic Database (NED), which is operated by the Jet Propulsion Laboratory, California Institute of Technology, under contract with NASA. Support for GO Program number 12592 was provided by NASA through a grant from the Space Telescope Science Institute, which is operated by the Association of Universities for Research in Astronomy, Incorporated, under NASA contract NAS5-26555.

Facility: HST(STIS)

\section{REFERENCES}

Arnett, W. D. 1982, ApJ, 253, 785

Badenes, C., Bravo, E., \& Hughes, J. P. 2008, ApJL, 680, L33

Bloom, J. S., Kasen, D., Shen, K. J., et al. 2012, ApJL, 744, L17

Bresolin, F. 2007, ApJ, 656, 186

Cardelli, J. A., Clayton, G. C., \& Mathis, J. S. 1989, ApJ, 345, 245

Foley, R. J. 2012, arXiv:1212.6261

Foley, R. J., Challis, P. J., Filippenko, A. V., et al. 2012a, ApJ, 744, 38
Foley, R. J., Filippenko, A. V., \& Jha, S. W. 2008, ApJ, 686, 117

Foley, R. J., \& Kasen, D. 2011, ApJ, 729, 55

Foley, R. J., Kromer, M., Howie Marion, G., et al. 2012b, ApJL, 753, L5

Foley, R. J., Simon, J. D., Burns, C. R., et al. 2012c, ApJ, 752, 101

Hamuy, M., Phillips, M. M., Suntzeff, N. B., et al. 1996, AJ, 112, 2391

Höflich, P., Wheeler, J. C., \& Thielemann, F.-K. 1998, ApJ, 495, 617

Horesh, A., Kulkarni, S. R., Fox, D. B., et al. 2012, ApJ, 746, 21

Howell, D. A., Sullivan, M., Brown, E. F., et al. 2009, ApJ, 691, 661

Jeffery, D. J., Leibundgut, B., Kirshner, R. P., et al. 1992, ApJ, 397, 304

Jin, Z., \& Gao, X. 2011, CBET, 2708, 1

Johansson, J., Amanullah, R., \& Goobar, A. 2013, MNRAS, 431, 43

Kelly, P. L., Hicken, M., Burke, D. L., Mandel, K. S., \& Kirshner, R. P. 2010, ApJ, 715,743

Lampeitl, H., Smith, M., Nichol, R. C., et al. 2010, ApJ, 722, 566

Lentz, E. J., Baron, E., Branch, D., Hauschildt, P. H., \& Nugent, P. E. 2000, ApJ, 530,966

Li, W., Bloom, J. S., Podsiadlowski, P., et al. 2011, Natur, 480, 348

Maguire, K., Sullivan, M., Ellis, R. S., et al. 2012, MNRAS, 426, 2359

Mandel, K. S., Narayan, G., \& Kirshner, R. P. 2011, ApJ, 731, 120

Margutti, R., Soderberg, A. M., Chomiuk, L., et al. 2012, ApJ, 751, 134

Mazzali, P. A., \& Podsiadlowski, P. 2006, MNRAS, 369, L19

Nugent, P., Sullivan, M., Bersier, D., et al. 2011a, ATel, 3581, 1

Nugent, P. E., Sullivan, M., Cenko, S. B., et al. 2011b, Natur, 480, 344

O’Donnell, J. E. 1994, ApJ, 422, 158

Park, S., Badenes, C., Mori, K., et al. 2013, ApJ, 767, 10

Patat, F., Cordiner, M. A., Cox, N. L. J., et al. 2013, A\&A, 549, 62

Perlmutter, S., Aldering, G., Goldhaber, G., et al. 1999, ApJ, 517, 565

Phillips, M. M. 1993, ApJL, 413, L105

Prieto, J. L., Stanek, K. Z., \& Beacom, J. F. 2008, ApJ, 673, 999

Richmond, M. W., \& Smith, H. A. 2012, JAVSO, 40, 872

Riess, A. G., Filippenko, A. V., Challis, P., et al. 1998, AJ, 116, 1009

Sauer, D. N., Mazzali, P. A., Blondin, S., et al. 2008, MNRAS, 391, 1605

Schlafly, E. F., \& Finkbeiner, D. P. 2011, ApJ, 737, 103

Shappee, B. J., \& Stanek, K. Z. 2011, ApJ, 733, 124

Silverman, J. M., Ganeshalingam, M., \& Filippenko, A. V. 2013, MNRAS, 430, 1030

Stoll, R., Shappee, B., \& Stanek, K. Z. 2011, ATel, 3588, 1

Stritzinger, M., Hamuy, M., Suntzeff, N. B., et al. 2002, AJ, 124, 2100

Sullivan, M., Conley, A., Howell, D. A., et al. 2010, MNRAS, 406, 782

Suzuki, N., Rubin, D., Lidman, C., et al. 2012, ApJ, 746, 85

Taubenberger, S., Hachinger, S., Pignata, G., et al. 2008, MNRAS, 385, 75

Timmes, F. X., Brown, E. F., \& Truran, J. W. 2003, ApJL, 590, L83

Tully, R. B., Rizzi, L., Shaya, E. J., et al. 2009, AJ, 138, 323

Walker, E. S., Hachinger, S., Mazzali, P. A., et al. 2012, MNRAS, 427, 103

Zhang, T., Zhou, Z., \& Wang, X. 2011, CBET, 2708, 3 